\title{
Developing a Methodology to Balance Benefit-Sharing: Application in the Context of Biodiversity Beyond National Jurisdiction
}

\author{
Jane Eva Collins *,a,b , Aysegul Sirakaya ${ }^{c, b}$, Thomas Vanagt ${ }^{\mathrm{b}}$ and Isabelle Huys $^{\mathrm{a}}$ \\ ${ }^{a}$ Faculty of Pharmaceutical Sciences, Clinical Pharmacology and Pharmacotherapy, Katholieke Universiteit Leuven, \\ Leuven, Belgium \\ ${ }^{b}$ ABSint, Bruges, Belgium \\ ${ }^{c}$ Department of European, Public and International Law, Faculty of Law, Ghent University, Ghent, Belgium
}

\begin{abstract}
The effectiveness and success of benefit-sharing measures to date, particularly in contributing towards the conservation and sustainable use of biodiversity, has been questionable. This is likely related to the degree of beneficial impacts versus burden on the users and regulatory authorities in terms of administrative complexities. It is, therefore, timely to reconsider which forms of benefit-sharing may most favourably balance the associated beneficial and burdensome aspects. The aim of this paper is to develop and demonstrate a benefit-sharing balance methodology which can be used as a tool to help decision-makers to select options in an objective and transparent manner. Application in the biodiversity beyond national jurisdiction context provides a useful example of how this tool can be used. Results suggest that sharing of genetic sequence data and research results provide the most favourable balance in terms of non-monetary benefit sharing, whilst the most favourable monetary benefit-sharing options were associated with research funding and salaries. The benefit-sharing balance methodology presented here provides a useful tool and starting point, which can be built upon in the future, to include more detailed information gathered from expert groups to consolidate the perceived balance of beneficial impacts versus burden. In addition, the equation can be tailored according to different policy settings where different benefit-sharing factors may be more appropriate. Ultimately, use of this tool could help to enhance implementation of benefit-sharing policies/legislation with greater potential to balance beneficial impacts with associated burden, thereby enhancing workability of the access and benefit-sharing system as a whole.
\end{abstract}

Keywords: benefit-sharing, methodology, genetic resources, marine, areas beyond national jurisdiction

Citation: Collins, J. E., Sirakaya, A., Vanagt, T., Huys, I. (2020). Developing a Methodology to Balance BenefitSharing: Application in the Context of Biodiversity Beyond National Jurisdiction. Genetic Resources 1 (1), $24-39$. doi: 10.46265/genresj.2020.1.24-39.

(C) Copyright 2020 the Authors.

This is an open access article distributed under the terms of the Creative Commons Attribution License (CC BY 4.0), which permits unrestricted use, distribution, and reproduction in any medium, provided the original author and source are

credited.

\section{Introduction}

According to Article 1 of the Convention on Biological Diversity (CBD, 1992), the objective of the Convention is 'the conservation of biological diversity, the sustainable use of its components and the fair and equitable sharing of the benefits arising out of the utilization of genetic resources ${ }^{11}$.

\footnotetext{
${ }^{*}$ Corresponding author: Jane Eva Collins

(jane.collins@abs-int.eu)

${ }^{1}$ Article 1, (CBD, 1992): 'The objectives of this Convention, to be pursued in accordance with its relevant provisions, are the conservation
}

Article 15.7 of the Convention takes a step further by stating that Parties shall take measures to share benefits such as results of research and development (R\&D) and benefits arising from commercial and other utilization of genetic resources (CBD, 1992). However,

\footnotetext{
of biological diversity, the sustainable use of its components and the fair and equitable sharing of the benefits arising out of the utilization of genetic resources, including by appropriate access to genetic resources and by appropriate transfer of relevant technologies, taking into account all rights over those resources and to technologies, and by appropriate funding.'
} 
despite attempts made by authors such as Morgera (2014), there is as yet no specific, internationally agreed legal definition of the term 'benefit-sharing' in the context of utilising genetic resources (Schroeder, 2007; Morgera and Tsioumani, 2010; Morgera, 2014; Parks, 2019). In addition, the terms 'fair' and 'equitable' are also not clearly defined (de Jonge, 2011; Morgera, 2014). This can lead to difficulties between stakeholder groups in terms of different interpreted definitions and requirements associated with benefit-sharing, as well as differences in motivation with respect to the notion (de Jonge, 2009, 2011). Nonetheless, the Nagoya Protocol and other genetic resource frameworks, such as the FAO International Treaty on Plant Genetic Resources for Food and Agriculture (ITPGRFA) and the WHO Pandemic Influenza Preparedness (PIP) Framework for the sharing of influenza viruses and access to vaccines and other benefits, provide suggestions of what benefitsharing could entail (FAO, 2001; Nagoya Protocol, 2011; World Health Organization, 2011). A list of types of nonmonetary and monetary benefits that can be shared are listed in the Annex of the Nagoya Protocol (2011).

Negotiations for a new international legally binding instrument (ILBI) under the United Nations Convention on the Law of the Sea (UNCLOS, 1982) for the conservation and sustainable use of marine biological diversity of areas beyond national jurisdiction (BBNJ) began in 2018. Negotiations address a 'package' of four elements and cross-cutting issues. The four elements include: marine genetic resources (MGR), including questions on the sharing of benefits; measures such as area-based management tools (ABMT), including marine protected areas (MPAs); environmental impact assessments (EIA); and capacity-building and transfer of marine technology (UNGA Res. 72/249, UN Doc. A/Res/72.249, 24 December 2017, para. 2.). In order to govern MGR in areas beyond national jurisdiction (ABNJ) and to answer questions on the sharing of benefits, it is important to consider which benefitsharing options may be most appropriate in this particular context.

Under the Nagoya Protocol, benefits should be shared by the users of genetic resources with the provider state, in accordance with domestic legislation (Art 5. Nagoya Protocol 2011). However, in the BBNJ context, although the types of users may be similar, there will be no provider of the genetic resources, since MGR exist outside the borders of national jurisdiction. It has not yet been agreed who the beneficiaries will be, but could involve 'developing states Parties, in particular least developed countries, landlocked developing countries, geographically disadvantaged states, small island developing states, coastal African states and developing middle-income countries' (Art 11, BBNJ draft text, 2019a). In addition, since the Nagoya Protocol applies only to areas within national jurisdiction, the same access and benefit-sharing (ABS) provisions do not apply to the utilisation of genetic resources from ABNJ. As such, new benefit-sharing options are under consideration for adoption as part of the new BBNJ agreement (BBNJ draft text, 2019a) ${ }^{2}$.

Whilst a list of potential benefit-sharing options can be found in the Annex of the Nagoya Protocol (2011), the effectiveness and success of these measures, and the ABS system as a whole, has been questioned by stakeholders (Fedder, 2013; Pauchard, 2017; RuizMuller, 2018). Between 1996 and 2017, a total of 217 ABS agreements for commercial research and 248 for non-commercial research were concluded (Pauchard, 2017). To date, there is a lack of evidence to support the assumption that benefit-sharing leads to effective conservation of biodiversity (Suneetha and Pisupati, 2009; Pisupati and Bavikatte, 2014). In addition, the burden on the users (such as universities and private companies) and regulatory authorities in terms of administrative complexity when complying with ABS legislation and conducting benefit-sharing can act as a disincentive for utilisation of genetic resources, potentially limiting the benefits derived and shared (Richerzhagen and Holm-Mueller, 2005; Tvedt, 2013). Further challenges exist with regards to achieving fair and equitable benefit-sharing and sustainable development (Louafi, 2013; Tsioumani, 2018). It is timely and appropriate, therefore, to reevaluate how the success of implementing benefitsharing options are measured and reconsider which forms may most fairly balance the positive and burdensome associated aspects (Ruiz-Muller, 2018). In order to understand this balance, key factors may be used to assess the overall positive influence of a benefitsharing option, such as the number of beneficiaries receiving benefits, the effect on biodiversity goals and the long-term impacts and significance of benefitsharing, as well as the overall burdensome aspects, such as the burden on the user of genetic resources and the burden on the regulator (Tvedt, 2013; Correa, 2017; Morgera, 2018a; Harden-Davies and Gjerde, 2019).

Consideration of the benefit-sharing options for adoption as part of the new BBNJ agreement provides a good opportunity to identify benefit-sharing factors and assess the overall positive versus burdensome balance of different benefit-sharing options. It has been noted that of the four elements under consideration as part of the BBNJ package, MGR including questions on the sharing of benefits remains the most challenging and immature element, with few detailed solutions suggested to date (Voigt-Hanssen, 2018). This is to be expected given the different stakeholder perspectives, goals and concerns Collins et al (2020). However, review of non-monetary and monetary benefit-sharing options in light of benefit-sharing factors and understanding of which measures may provide the most fair and balanced outcome could provide a useful platform on which negotiatons can progress (BBNJ, 2019a; Sirakaya, 2019; Collins et al, 2020).

\footnotetext{
${ }^{2}$ See Part II, Article 11 '[Fair and equitable] sharing of benefits', Draft BBNJ Agreement, 2019a.
} 
The first aim of this paper, therefore, is to develop a benefit-sharing methodology which can be used as a tool to understand the balance of beneficial versus burdensome aspects associated with different benefitsharing options. This will involve making use of a multi-criteria analysis technique (MCA) to help decisionmakers to select options in an objective and transparent manner (de Brucker et al, 2013). The main objective of the MCA technique is to overcome challenges that human decision-makers experience when handling large amounts of complicated information in a consistent manner (Dodgson et al, 2009). The second aim is to demonstrate how the methodology can work by applying it to the BBNJ context, to review the different types of non-monetary and monetary benefit-sharing options with potential for adoption under the new agreement, in order to determine which forms may provide more balanced outcomes. The BBNJ context was selected for demonstration of the methodology because these negotiations represent a relevant, current opportunity which may directly benefit from such an exercise with regards to making informed decisions in terms of the benefit-sharing options to be adopted in the treaty text. This objective was achieved by identifying the key benefit-sharing factors to consider, as well as their relative importance to stakeholders. The benefitsharing factors were then applied to different benefitsharing options through the use of the new equation, to reveal the balance of beneficial impacts versus burden associated with the different options.

The authors acknowledge the limited number of interviewees involved and suggest that further interviews with a larger number of participants would be needed to draw significant conclusions in the context of ongoing BBNJ negotiations, as well as also in other genetic resource ABS circumstances. In addition, the authors wish to highlight the broad scope of the current paper and general nature of the factors considered. Future research is needed to build on the results gathered in this study and to include more comprehensive literature review as well as more detailed information gathered from various expert groups. This would help to consolidate the perceived balance of beneficial impacts versus burden associated with benefit-sharing options.

\section{Development of a Benefit-Sharing Tool}

\section{Materials and Methods}

The study began with a scoping literature review to identify benefit-sharing factors that may enable analysis of the positive versus burdensome aspects of benefitsharing options. The benefit-sharing options which could be considered for a potential governance (ABS) system for MGR from ABNJ were also identified (FAO, 2001; Nagoya Protocol, 2011; World Health Organization, 2011; BBNJ, 2019b; Collins et al, 2020). The literature review involved searches through Pubmed, Embase, EurLex, the United Nations Convention on the Law of the Sea, the United Nations BBNJ website ${ }^{3}$, the Convention on Biological Diversity (CBD) and the Nagoya
Protocol (UNCLOS, 1982; CBD, 1992; Nagoya Protocol, 2011). Search keywords included: benefit-sharing, ABS, genetic resources, MGR, ABNJ, and capacity building.

\section{Results}

\section{Benefit-Sharing Factors}

As a result of the authors' own experience in terms of how benefit-sharing works in practice, specifically the modalities of drafting and negotiating benefitsharing contracts, coupled with literature review, five factors were identified as being the main objectives and considerations of benefit-sharing associated with utilisation of genetic resources (referred to in this paper as 'benefit-sharing factors', Young and Tvedt, 2017). Listed below and in Table 1 are descriptions of the benefit-sharing factors considered in this study.

1. Biodiversity goals: Different goals and objectives, as defined by legal biodiversity acts or treaty's, may be attained through benefit-sharing. BBNJ context: relevant goals include contributing towards conservation of marine biological diversity of $A B N J$, promoting sustainable use of MGR from $A B N J$, fostering scientific $R \& D$ and promoting fair and equitable benefit-sharing (Art 140, UNCLOS, 1982; CBD, 1992; Harden-Davies and Gjerde, 2019; Collins et al, 2020).

2. Direct beneficiaries: The number of individuals receiving benefits will vary according to the type of benefit that is being shared. BBNJ context: a greater number of individuals may have access to MGR data if this is shared, such as via online databases, compared to the number of people who may receive funding to conduct $\mathrm{PhD}$ projects on topics related to BBNJ.

3. Benefit-sharing significance: The impact associated with different forms of benefit-sharing could be approximately determined in terms of the significance, value and the duration of the impact. For example, impacts could include enhanced employment and contribution towards scientific knowledge. Duration of impacts may vary from weeks to months or years and could give an indication of whether future generations may be positively influenced by the benefit-sharing or not (HardenDavies and Gjerde, 2019). BBNJ context: training courses as part of capacity building initiatives may lead to longer-term positive impacts on a group of people, perhaps linked to enhanced employability, than would be the case for access to MGR data.

4. Burden on the user: Users could include any organisations or individual researchers from developed or developing states. If benefit-sharing is a requirement, these users may encounter a degree of burden linked to the process of sharing. This burden may take the form of monetary cost to

\footnotetext{
${ }^{3}$ https://www.un.org/bbnj/ - accessed 08 March 2020
} 
generate the so-called 'benefits' in the first place, such as tailored training courses or a monetary payment, but also the transaction cost of sharing benefits. In addition, the generation and sharing of benefits will involve human labour costs, for example sending experts to beneficiary locations to conduct capacity-building or training initiatives. Time may represent another burdensome aspect. Whilst some types of benefit-sharing require only one simple transaction, other forms may require repeated actions over time. BBNJ context: sharing of benefits in the BBNJ context may involve burdens and costs to the user, for example to set up and organise databases in which MGR data can be stored and accessed, or sending of laboratory equipment as a form of technology transfer.

5. Burden on the regulator: A regulator may encounter a degree of burden linked to the process of benefit-sharing, as well as related to the need to build capacity in the context of novel regulation. This may involve raising awareness of and enforcing compliance with applicable legislation. These burdens may be both in terms of monetary costs and human labour costs, similar to those described for the users above. However, for the regulator, these costs may be linked to monitoring and checking compliance of users with the benefit-sharing requirements. BBNJ context: at present, it remains unclear whether there will be a regulator for $\mathrm{ABNJ}$, or who this could be (Mohammed, 2017; BBNJ, 2019b). However, in the event that there is a regulator/regulatory body charged with regulating ABS linked to MGR from $A B N J$, and benefit-sharing is a requirement, then the regulator may face burdens associated with overseeing the process of benefit-sharing, particularly in terms of monetary costs, complexity and time.

\section{Rationale for Selecting These Five Benefit-Sharing Factors}

The reasoning behind the five factors on benefit-sharing is threefold. The first reason relates to the concept of establishing a balanced ABS system as referred to in our previous research (Sirakaya, 2019, 2020). For an ABS system to successfully establish balance between the user and the provider (in bilateral genetic resources frameworks), so as to fairly distribute benefits arising from utilisation of genetic resources between the users of genetic resources and beneficiaries, it is crucial to ensure that such a system attends to the international biodiversity goals. The authors here specifically explore benefit-sharing options and therefore the factors pay due regard to the international biodiversity goals as identified under our current research as well as the previous research conducted on the matter (Sirakaya, 2020).

Secondly, the five factors were selected since they clearly define and influence the modalities of benefit- sharing and can be negotiated when drafting agreements (Young and Tvedt, 2017). The authors believe that these factors, coupled with agreement between users and providers, can promote an objective balance in benefit-sharing.

Multiple stakeholder groups are involved within the BBNJ context and it is of utmost importance that an instrument is developed that attains the needs of all of these groups to the greatest extent possible. A stakeholder-driven MCA is the best available method to achieve this (de Brucker et al, 2013) (Sirakaya and De Brucker, personal communication). A recent study conducted by Sirakaya and De Brucker (under review) demonstrates how MCA can been applied to design regulatory frameworks for access to genetic resources in cases which involve multiple stakeholders. As such, the third reason for the selection of the criteria refers to the importance of stakeholder consultation under the MCA. According to Freeman (1984), stakeholders are defined as 'any individual or group who can affect an organisation's performance or who is affected by the achievement of this organisation's objectives.' Looking into regulatory issues related to benefit-sharing in ABNJ, taking into account Freeman's definition, five key stakeholder groups were identified: developing states, developed states, civil society, the scientific research community and the private sector (Collins et al, 2020). Although questions remain regarding whether there will be any form of regulatory body in the BBNJ context, this could potentially involve members from both developing and developed states working together to fulfill the role. The scientific research community and private sector represent the potential users of MGR. Developing states and civil society are the likely beneficiary groups who would receive benefits shared from utilisation of MGR from ABNJ.

Stakeholders' perceptions play a crucial role in MCA conducted on ABS frameworks. This is due to the fact that a balanced $A B S$ system can only be established if there is a reasonable ABS framework that the users would be incentivised to adhere to. The same principle applies to a potential benefit-sharing system for ABNJ. The users (i.e. the scientific research community and private sector) would only be incentivised if there is a foreseeable balance between the impact of being a part of the system and the burden thereof. Likewise, the system would only function as intended if the cost borne to the regulator (including members from developing and developed states) of establishing and maintaining the system is considerably lower than the benefits generated through the system to be shared with beneficiaries (developing states and civil society). In line with this, Harden-Davies and Gjerde (2019) stipulate the 'need to strike a balance between the right to use and the responsibility to share'. Therefore, by reviewing the balance of potential beneficial impacts (private, social and environmental aspects) versus burden, negotiators may be in a better position to make informed decisions regarding which benefit-sharing options may be most 
Table 1. Description of the five benefit-sharing factors considered in this study.

\begin{tabular}{|c|c|c|}
\hline Benefit-Sharing Factors & Description & References \\
\hline Biodiversity goals (G) & $\begin{array}{l}\text { i. Contributing towards conservation of marine } \\
\text { biological diversity of ABNJ } \\
\text { ii. Promoting sustainable use of MGR from ABNJ } \\
\text { iii. Fostering scientific R\&D } \\
\text { iv. Promoting fair and equitable benefit-sharing } \\
\text { v. Inclusivity of developing states in access to and } \\
\text { utilisation of MGR of ABNJ }\end{array}$ & $\begin{array}{l}\text { CBD (1992); Collins et al } \\
\text { (2020); BBNJ (2019a); } \\
\text { Harden-Davies and Gjerde } \\
\text { (2019); Morgera (2018a); } \\
\text { UNCLOS (1982) }\end{array}$ \\
\hline Direct beneficiaries (\#) & $\begin{array}{l}\text { i. Number of individuals impacted/receiving the } \\
\text { benefits }\end{array}$ & Morgera (2014) \\
\hline Benefit-sharing significance (S) & $\begin{array}{l}\text { Size of impact, for example: } \\
\text { i. Duration (months or years - gives an indication of } \\
\text { whether the impact will be beneficial to current and } \\
\text { future generations) } \\
\text { ii. Enhanced employability/employment rates } \\
\text { iii. Meaningfulness }\end{array}$ & $\begin{array}{l}\text { Harden-Davies and Gjerde } \\
\text { (2019) }\end{array}$ \\
\hline Burden on the users of (M)GR (U) & $\begin{array}{l}\text { i. Monetary cost and human labour to generate and } \\
\text { share the benefits, including costs to transfer to and } \\
\text { distribute amongst beneficiaries. } \\
\text { ii. Opportunity cost } \\
\text { iii. Time value }\end{array}$ & $\begin{array}{l}\text { Harden-Davies and Gjerde } \\
\text { (2019) }\end{array}$ \\
\hline Burden on the regulator $(\mathrm{R})$ & $\begin{array}{l}\text { i. Monetary cost and human labour to monitor } \\
\text { benefit- sharing } \\
\text { ii. Complexity } \\
\text { iii. Time value }\end{array}$ & Pisupati and Bavikatte (2014) \\
\hline
\end{tabular}

appropriate to consider including as part of a new benefit-sharing framework for ABNJ.

In sum, these five benefit-sharing factors are crucial in establishing a balanced benefit-sharing framework for ABNJ with governance options that attain international biodiversity objectives, as outlined by legal acts and treaty's, and pay due regard to stakeholder preferences (Collins et al, 2020).

\section{Benefit-Sharing Balance}

In order to objectively evaluate different benefitsharing options in terms of the ratio of potential positive impacts versus associated burden in a fair and consistent manner, an equation was proposed, taking into consideration the five benefit-sharing factors (see Figure 1). Weighted values were used in this study to incorporate consideration of the fact that some benefitsharing factors may be perceived as more important than other factors and should therefore contribute more to the final score. Different methods can be used to generate the weighting values for different factors. For example, an analytic hierarchy process, case-based reasoning, simple multi-attribute rating technique, mathematical programming or interview approaches could be used (von Winterfeldt and Edwards, 1986; Ho et al, 2010; Nerini et al, 2014). In this study, the values of weightings were obtained through an interview process. Benefit-sharing factors ( $G, \#, S, U, R)$ were first each multiplied by an average weighting (a-e) assigned to each factor by interviewees. The three positive, weighted benefit-sharing factor scores are multiplied together, as are the two burdensome, weighted factor scores. The positive aspects are then divided by the burdensome aspects to give a benefit-sharing balance value for each benefit-sharing option.

\section{Applying the Benefit-Sharing Tool to the BBNJ Context}

\section{Materials and Methods}

After development of the benefit-sharing methodology (see Figure 1), a semi-structured, qualitative interview was prepared (see Supplemental File 1). Interviews were conducted with ten experts. Availability and willingness to participate in the interview represented a controlling factor in the recruitment of participants, as well as the variety of stakeholder groups, and contributed to the limited numbers.

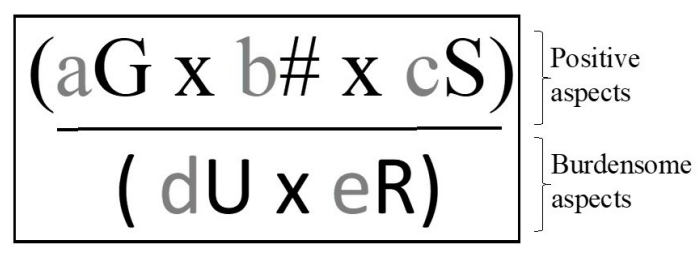

Figure 1. Equation to determine the balance of potential positive impact versus burden associated with different forms of benefit-sharing. Letters a-e represent the average weight assigned by interviewees to the five benefit-sharing factors. Benefit-sharing factors: $\mathrm{G}=$ biodiversity goals; $\#=$ direct beneficiaries; $\mathrm{S}$ = benefit-sharing significance; $\mathrm{U}=$ burden on the user, and; $\mathrm{R}=$ burden on the regulator. 


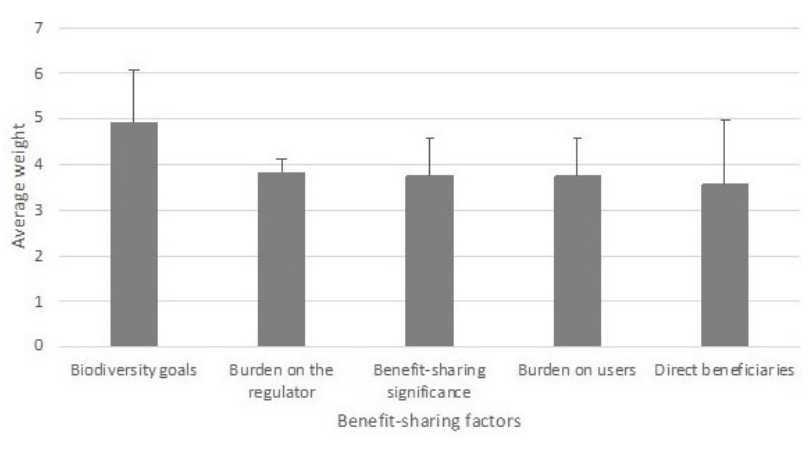

Figure 2. Average weight assigned by interviewees to the five different benefit-sharing factors considered. Error bars indicate standard deviation of the mean.

These experts are based in different locations around the world (four from developing and six from developed states) and representative of different stakeholder groups: two from the scientific research community (one from a developed and one from a developing state), three from developing states delegations, three from developed states delegations, and two from civil society (both from developed states). Whilst effort was made to include private sector stakeholders in the interview process, and indeed representatives from one organisation did provide helpful feedback on some of the questions, time constraints and availability of representatives rendered this not possible in the scope of the current study. Interviews were conducted during the third session of the intergovernmental conference (IGC3) for BBNJ in New York (between 19-28 August 2019).

The interviews followed a pre-defined guide list of questions (see Supplemental File 1), which started by requesting participants to rank five benefit-sharing factors (see Table 1) on a scale from 1 to 5 in terms of perceived relative importance (see Figure 2). Participants were then asked to score a variety of nonmonetary and monetary benefit-sharing options in terms of potential impact of these on the five benefit-sharing factors (from $0=$ no impact, to $5=$ very high impact), according to their perspective, and to give short reasons for their decisions. These scores were used to perform a MCA, whereby scores were multiplied by the average weighting assigned to each factor and inserted into an equation (see Figure 1), to determine the balance of potential positive impact versus burden associated with each different form of benefit-sharing (see Figure 3 and Figure 4). This enabled comparison of the ratio of potential positive impacts versus burden between different benefit-sharing options, taking into account the relative importance of different key factors. Interviews were audio-recorded and transcribed. Microsoft Excel software was used as a means to store the data. A thematic analysis of the transcripts was conducted to identify common themes in responses. All data were anonymised by grouping results into stakeholder groups. Written informed consent forms were signed by all of the interviewees in this study.

\section{Results}

\section{Weighting the Benefit-Sharing Factors}

According to the average stakeholder ranking, biodiversity goals were considered as the most important factor to be taken into consideration when assessing how balanced different benefit-sharing options are. The other four benefit-sharing factors were considered approximately equal in terms of importance (see Figure 2). The range of ranking given by interviewees was greatest for the direct beneficiaries factor, as indicated by the larger error bar in Figure 2. Reasons given by interviewees for these rankings are described below.

Biodiversity Goals. The majority of interviewees stated that the biodiversity goals are the most critical factor from their perspective. Whilst many different goals could fall under this category, interviewees most frequently referred to conservation of biodiversity as the aspect that they consider most important. This is because the goals of 'conservation and sustainable use of marine biological diversity' are the key overarching goals of the new agreement as a whole (BBNJ, 2019b). Collins et al (2020) present further detailed information regarding stakeholder goals for a potential new ABS mechanism for ABNJ. Other goals, such as promoting marine scientific research, are viewed as necessary to achieve the conservation objectives. They also indicated that biodiversity goals are more important than any of the other four benefit-sharing factors considered in this study. One interviewee noted that biodiversity goals are important for all stakeholders involved, not just for the beneficiaries of benefit-sharing.

Direct Beneficiaries. Most interviewees indicated the benefits should be shared amongst as many people as possible, and that the greater the number of people who are positively affected the better. However, three interviewees also noted that the importance of beneficiaries depends on the definitions, whether it is only the people who are directly affected, or also those who indirectly benefit. Sometimes only a few people may immediately and directly benefit from a benefitsharing initiative, such as collaborative or joint venture projects, but such an effort may indirectly have a large impact on many more people and other factors, perhaps over time. For example, sharing of MGR samples and data for scientific research may only initially affect a moderately low number of people, but if this leads to development of new pharmaceutical products to treat human illness or to maintaining the health of the ocean through conservation measures, then a far larger number of beneficiaries will be encountered.

Benefit-Sharing Significance. The majority of interviewees assigned this factor a moderately high score. Two interviewees stated that the duration of benefit-sharing significance is vital. However, two other interviewees gave this a moderately low score and suggested that, from their perspective and in the current context, this factor was not as important as the others considered in this study. 


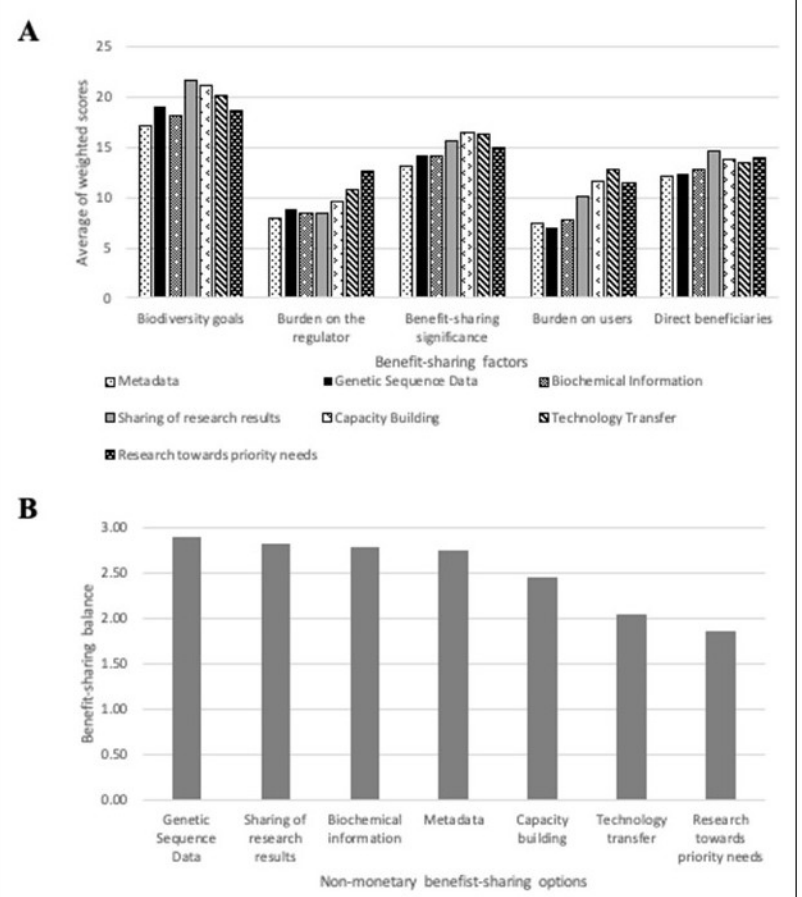

Figure 3. A) Average weighted scores for the seven nonmonetary benefit-sharing options according to the five benefitsharing factors, and B) scores according the benefit-sharing balance equation for the seven non-monetary benefit-sharing options.

Burden on the Users of (M)GR. Burden on the user was considered moderately important by over half of interview participants. One interviewee raised the question of whether there might be the possibility to charge a 'handling fee' for certain types of benefits (such as sharing of material). The degree of burden on the user may be affected by whether there is the possibility to charge a handling fee (or similar) to disseminate MGR samples and other benefits. It was suggested that sharing of benefits cannot all be for free.

Burden on the Regulator. The majority of interviewees assigned this factor a high score. Whilst it remains unclear whether there might be or who might constitute the regulatory authority in the BBNJ context, it is possible that such an institution will be established. Interviewees acknowledged that this is an important factor because if burden on the regulator is not taken into careful consideration, then the whole benefitsharing system, whether non-monetary or monetary, could fail. For example, if the system is overly expensive, then any potential monetary benefits may be used to fund running of the system itself rather than accruing for the benefit of beneficiaries (Morgera and Tsioumani, 2010; Morgera, 2014; Tsioumani, 2018). In addition, burden on the regulator could result in lengthy decisionmaking processes, thereby indirectly affecting potential users.

\section{Non-Monetary Benefit-Sharing Options}

To gather perspectives on benefit-sharing, interviewees were asked to consider the potential influence of different benefit-sharing options on the five benefit- sharing factors (Table 1). Analysis of the President's aid to negotiations (2019) and a review of the literature lead to identification of seven non-monetary benefitsharing options which could be considered in the context of governing the utilisation of MGR from ABNJ (see Figure 3). A description of the options considered in this study, and their significance, can be found in Sirakaya (2019) and Collins et al (2020). Non-monetary benefitsharing options considered here include:

\section{Sharing of Raw Data:}

(a) Metadata;

(b) Genetic Sequence Data (GSD) ${ }^{4}, 5,6$

(c) Biochemical Information

2. Sharing of Research Results

3. Capacity Building

4. Technology Transfer

5. Research Directed Towards Priority Needs

The benefit-sharing balance, as calculated using the equation described above, indicated the most favourable balance of beneficial impacts versus burden associated with sharing of GSD and sharing of research results. Sharing of biochemical information, metadata and capacity building received a similar, but slightly lower score. Technology transfer, and in particular research directed towards priority needs, received less favourable balances (see Figure 3B). Reasons given by interviewees for potential positive impacts versus burden associated with different non-monetary benefit-sharing options are described below.

Sharing of Raw Data (Metadata, GSD and Biochemical Information). For some participants, all three types of raw data sharing are viewed together as a package. It was suggested that if raw data were to be made publicly available online, this could have a positive impact on a relatively large number of beneficiaries (Figure 3A). This is because many people would then be able to access the data easily, quickly and possibly for free. However, two interviewees also acknowledged that many people, particularly in developing states, may not be able to make use of the raw data since they lack the capacity to work on it. As such, unless accompanied by capacity building, the number of beneficiaries impacted by sharing of raw data may not actually be very high.

Interviewees suggested that sharing of raw data may not immediately have a large effect on the number of

\footnotetext{
${ }^{4}$ GSD is the term most commonly used in the BBNJ context to refer to data/information which is described as Digital Sequence Information (DSI) under the auspices of the CBD. According to the Consortium of European Taxonomic Facilities (CETAF) and the Society for the Preservation of Natural History Collections (SPHNC), the term GSD is in line with the concept of DSI. However, despite efforts to define DSI/GSD, there is currently no official, internationally accepted definition of the term.

${ }^{5}$ https://www.cbd.int/abs/DSI-views/2019/CETAF-DSI.pdf accessed 09 March 2020

${ }^{6}$ https://www.cbd.int/abs/DSI-views/2019/SPNHC-DSI.pdf accessed 09 March 2020
} 
direct beneficiaries, the biodiversity goals or benefitsharing significance. However, by building up large data sets, the beneficial impact of sharing raw data on these benefit-sharing factors may grow and could become relatively high over time. This could also be in part due to the long-lived nature of data; once data is curated and stored, it can be made available and accessed for a very long time. One interviewee suggested that sharing of raw data could be one of the best things we could do to have a positive influence on the benefit-sharing significance.

One interviewee explained that a lot more work is required to generate biochemical information than for generation of metadata and GSD, because the process is comparably less straightforward. In addition, one interviewee suggested that whilst it is already best practice to share metadata and GSD, they were unsure whether it is yet best practice to share all biochemical information. Therefore, the burden on the user associated with sharing of biochemical information, in terms of opportunity cost, may be higher than that associated with the other forms of raw data sharing. In addition, the burden on the users related to sharing of data may depend on the stakeholder group in question and the type of data that is required to be shared. For some users, such as those in the private sector, this is likely to be very burdensome and possibly even a deterrent, particularly if mandatory and involves the obligation to share commercially important data. This is because mandatory sharing of raw data would likely change the incentive to invest. Scientists in the private sector routinely conduct novel scientific research and publish results in scientific journals. They also disclose scientific information in the form of patents. However, from a commercial viewpoint, if sharing of data beyond current practice is mandatory, this may have broad implications for protection of intellectual property (IP) and for maintaining competitive market advantage. As such, mandatory sharing of valuable data may disincentivise investments in private sector projects involving MGR from ABNJ.

Interviewees indicated that the level of burden on the users and on the regulators would depend on the way in which benefit-sharing is done. Under the assumption that a new system is developed and must be used to share benefits, for example sharing of GSD through a clearing house mechanism or other form of database, this could lead to significant burden on the users and regulators. One interviewee noted that in the draft treaty text (BBNJ, 2019a), reference is made to a new system whereby benefits will need to be shared through a clearing house ${ }^{7}$ and raises the question of timing.

\footnotetext{
7 'Samples, data and related information shall be made available in open access [through the clearing-house mechanism [upon access] [after [...] years]]' and 'States Parties shall publish and communicate the reports of the results of the assessments in accordance with [articles 204 to 206] [article 205] of the Convention [, including through the clearing-house mechanism]' (President's aid to negotiations, 2019).
}

According to the interviewee, the issue of when and how benefits will be shared could have huge impacts on the degree of burden experienced. If benefit-sharing is left according to current practices, particularly in terms of where raw data are stored, then this could have very little or even zero impact on the burden felt by users or the new regulator. However, if we proceed with a new system, requiring a change in the way that data are shared, burden may be encountered by users in terms of requiring conversion of data into a particular format to fit into a specific database. As such, if benefitsharing were to become mandatory and involves new requirements and procedures, the burden on the users will be higher than at present.

The burden on the regulator could be relatively straightforward, if all they have to do is verify that data is being shared with a database, but the degree of burden will depend on how much information they need. One interviewee also noted that it may be important to consider the burden on the beneficiaries accepting raw data. This may involve requirements to develop infrastructure to receive and make use of data.

Sharing of Research Results. Interviewees indicated that a relatively large number of beneficiaries could be impacted by the sharing of research results (Figure 3A). However, one interviewee suggested that research results, such as a research paper, may be read by fewer people compared to the number of people who could access or use raw MGR data.

Sharing of research results could have a large positive effect on the overall impact of benefit-sharing, and also on the biodiversity goals such as conservation. It was suggested that this could be partly due to the long-lived nature of research results.

Interviewees suggested that the impact of sharing research results on the burden experienced by users would be moderate, since writing of research articles inherently involves a degree of human labour costs. However, it was also noted that this could be less burdensome for users than sharing of raw data, because this would involve a different system which may remain more similar to current practice. It was suggested that the burden on the regulator may also be moderate, but would depend on whether this was mandatory or voluntary. If the regulator was required to track that sharing of research results has been done, then this could lead to quite a heavy burden.

Capacity Building. It was suggested that the influence of capacity building on biodiversity goals and significance would depend on how sustainable the capacity building initiatives are. For example, if scientists are trained as part of a capacity building effort and then decide to leave the country, this cannot be considered as sustainable. According to one interviewee, this is unfortunately the case when reviewing current capacity building activity, and represents a challenge faced in many developing countries. The key question here is how to ensure the sustainability of capacity building. In an ideal 
world capacity building would be sustainable, so that effects are long-lasting.

The burden on the users of MGR may be greater in association with capacity building than some of the other options (Figure 3A). This is because it requires a combination of time, money and specific expertise to execute such an initiative.

Technology Transfer. It was suggested that the number of beneficiaries who could benefit from technology transfer would be moderately low. It was proposed that this may be because only a few people will be able to make use of the technology, particularly if the technology is very sophisticated and the absorptive capacity is low. One interviewee suggested that the impact of technology transfer on the biodiversity goals will be less than that associated with other benefitsharing options (Figure 3A). As with capacity building, the influence of technology transfer on the benefitsharing significance will depend on the sustainability. Interviewees were of the opinion that technology transfer may have an impact for a short amount of time, but were uncertain whether this would have long-lasting effects.

The burden on the users related to technology transfer may depend on the stakeholder group in question, the type of technology that is required to be shared, who pays for the transfer of technology and the means by which this is done. It was suggested that for some users, such as the scientific research community, this may be less burdensome than sharing of raw data. However, for other uses, such as those in the private sector, this is likely to be very burdensome, particularly if mandatory. This is because investors in the private sector aim to recoup investments, and mandatory, free transfer of technology would possibly discourage further investment. Alternatively, one interviewee suggested that if technology transfer were to be conducted on commercial terms, this could represent less burden for the private sector.

Research Directed Towards Priority Needs. One interviewee stated that, although research directed towards priority needs has the potential to reach a lot of people and could have a very high impact on attending to the biodiversity goals, particularly contributing towards conservation and sustainable use, they simply did not think that it was going to happen. This is because the interviewee felt that such a system of focusing research on priority needs would be unfeasible and unworkable. As such, the anticipated number of beneficiaries and influence on biodiversity goals and significance was scored relatively low (Figure 3A). In addition, it was noted that the impact of research directed towards priority needs on the benefit-sharing factors would depend on what exactly the priority needs are.

The burden on the user was thought to be moderately high, unless the users are already conducting research in the priority needs area. One interviewee suggested that such priority needs could be linked to research with socially beneficial uses, including 'health and food security' as described in Article 8 of the Nagoya Protocol (2011). This is because forcing scientists to change their research from one field of work to a different one, associated with the identified priority needs, would require significant monetary costs. Similarly, the burden on the regulator would likely also be high. It was suggested that the regulator may experience burden associated with understanding who the developing states are, identifying their priority needs and deciding how this should be regulated. One interviewee noted that it would likely be very difficult for a regulator to monitor this, and could be very challenging if it involves a new system in which to direct their regulation towards this specific purpose.

\section{Monetary Benefit-Sharing Options}

Analysis of the President's aid to negotiations (2019) and a review of the literature lead to identification of eight monetary benefit-sharing options which could be considered in the BBNJ context (see Figure 4). A description of the options considered in this study, and why they might be important, can be found in Sirakaya (2019) and Collins et al (2020). Monetary benefitsharing options considered here include:
1. Research Funding
2. Salaries
3. Joint Ventures ${ }^{8}$
4. Joint IP Rights
5. Royalties
6. Milestone Payments
7. License Fee
8. Access Fee

Interviewees were asked to consider the potential impact of different monetary benefit-sharing options on the five benefit-sharing factors. According the benefit-sharing balance calculation, the most favourable monetary benefit-sharing option, in terms of balancing beneficial impacts versus burden, was research funding followed closely by salaries (see Figure 4B). Joint ventures were considered the next most favourable option, followed by joint IP rights. Access fee, milestone payments, license fee and royalties were viewed as similar in terms of balancing the potential positive impacts versus burden. Reasons given by interviewees for potential positive impacts versus burden associated with different monetary benefit-sharing options are described below.

Interviewees noted that their answers in this section were given under the assumption that commercialisation of MGR is successful and that there will be money to share. This is crucial, because four interviewees out of the ten indicated that they do not believe that there will be any financial profits derived from R\&D on MGR, and therefore no money to share.

\footnotetext{
${ }^{8}$ In this paper, the term 'joint ventures' is used in a general, broad sense to describe a collaborative project/initiative undertaken jointly by two or more entities which otherwise retain their distinct identities. The term is not used here in the corporate or legal manner.
} 


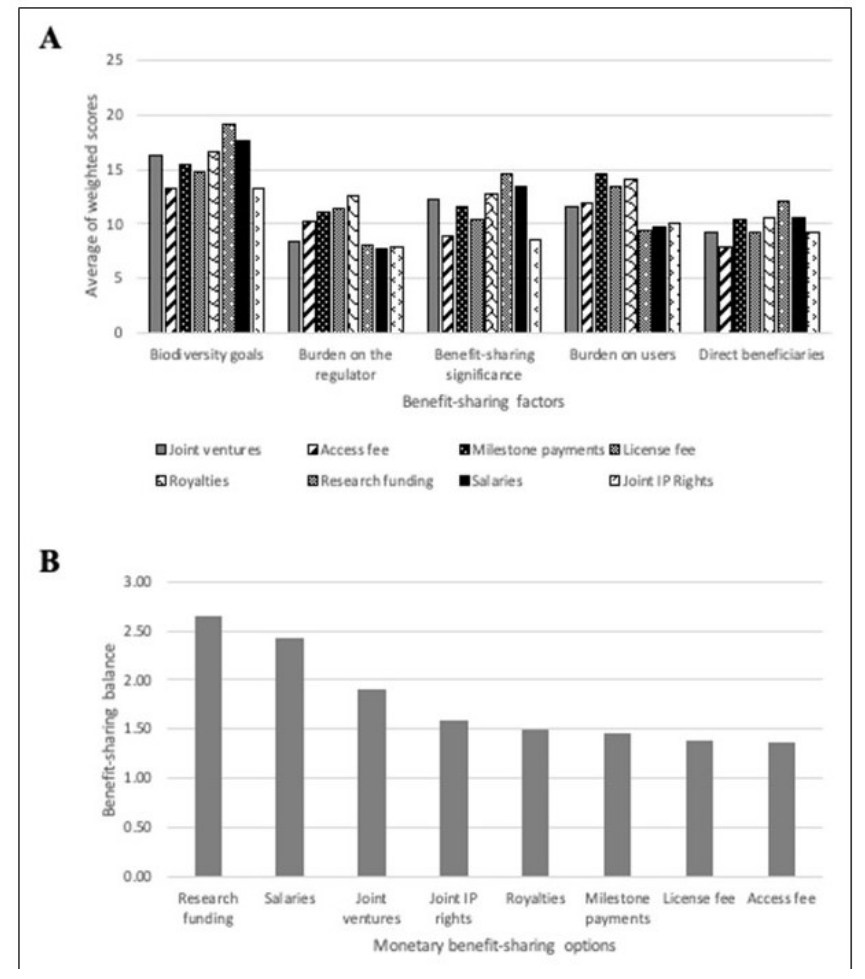

Figure 4. A) Average weighted scores for the eight monetary benefit-sharing options according to the five benefit-sharing factors, and B) scores according to the benefit-sharing balance equation for the eight monetary benefit-sharing options.

Research Funding and Salaries. Five of the ten interviewees indicated that they view research funding and salaries as similar in terms of the potential positive impacts versus burden. Interviewees indicated that research funding and salaries could have a greater positive influence on the biodiversity goals and benefit-sharing significance than the other forms of monetary benefit-sharing, and may have more longterm positive effects (see Figure 4A). For example, research funding could help to build capacity in developing states, potentially contributing towards enhanced employability, and could also be directly focused on long-term conservation and sustainable use of biodiversity.

One interviewee noted that the level of burden on the user associated with research funding and salaries would depend on who is paying for it. The burden on the users would be low if this is to be paid by the regulator, and the burden on the regulator may be relatively high. However, vice versa may be true if the users are required to pay this. It was also suggested that since research funding is something that is already done by the scientific community, then the burden on the regulator would likely be less for research funding than other monetary benefit-sharing options, if the regulator is only required to make sure that the money has been channeled appropriately.

Joint Ventures. Most interviewees agreed that joint ventures would influence a relatively low number of beneficiaries (Figure 4A). One participant stated that joint ventures will require money which cannot be shared amongst many people, and therefore the number of people affected will likely be low. The influence of joint ventures on the biodiversity goals and significance was also thought to be low, and would likely depend on the conditions.

It was suggested that the level of burden experienced by the user and the regulator would be moderate, but could be low if joint ventures were considered on a voluntary rather than a mandatory basis. This is because if joint ventures were required on a mandatory basis, then perhaps users/ organisations who are not equipped with the appropriate capacity would struggle to establish such projects.

Joint IP Rights. The majority of interviewees felt that joint IP rights (IPR) would impact a low number of beneficiaries (Figure 4A). One interviewee suggested that perhaps it was possible for this number to grow over time. Participants were also of the view that the influence of joint IPR on biodiversity goals and benefitsharing significance would be low, possibly with no longterm positive effects. With regards to burden on the user, interviewees suggested that this would depend on the conditions associated with the joint IPR, but was likely to be moderate to high. The burden on the regulator is likely to be high, and participants noted that they were unsure how this form of benefit-sharing could be regulated.

One interviewee indicated that they did not see joint IPR as a feasible option, as it would either not be relevant or appropriate in most cases. The interviewee stated that only inventors or co-inventors can claim IPR and requesting joint ownership with entities who do not represent this would lead to an inability to fulfill the required criteria to claim IPR. The only situation where such an approach has been considered previously is in the context of traditional knowledge, where indigenous knowledge may be included in an invention. However, according the interviewee, even then there are much better approaches to follow than joint IPR in order to share benefits. Nonetheless, if scientists from developing countries are included in a research project and at some point in time a new invention is developed, then joint IPR could be appropriate. It was also noted that publications have copyrights involved, which could also be considered as a form of joint IPR if multiple coauthors are involved in the effort. As such, the feasibility of these options will depend on the details regarding how this is done.

Milestone Payments, License Fee and Royalties. Most interviewees considered that milestone payments, license fees and royalties would lead to similar impacts on the benefit-sharing factors (see Figure 4A). One reason given for this is that people find it very difficult to distinguish between the three. It was suggested that the milestone payments could have similar positive impacts on the benefit-sharing factors as an access fee (see below). As with the access fee, questions were raised regarding whether users of MGR would be able to pay the milestone payments, license fees or royalties. One 
interviewee stated that they did not believe that a license fee would actually be workable.

Interviewees indicated that license fees and royalties may be more burdensome on the user and regulator than the milestone payments, but that all three would likely be more burdensome than an access fee. This is because the access fee could take the form of a one-off payment (with no subsequent costs), of a preset and limited amount, whereas the others may incur more than one fee and may be payable at times which are less clearly defined than at the point of access (Collins et al, 2020).

Access Fee. The majority of interviewees were of the opinion that an access fee would impact only a low number of beneficiaries (Figure 4A). It was suggested that this may be in part because relatively little money would be generated and few users would be able to pay this if it was expensive, although research institutions could potentially seek scholarships to cover this (see Collins et al, 2020) for more detail). Questions were raised regarding to whom this fee would be paid.

The influence of an access fee on the biodiversity goals and significance was also considered low. One interviewee noted that the influence would depend what this fee is used for, but was sceptical that it would have a positive effect on these factors. Another interviewee stated that an access fee system could be attractive because it would ensure that some income is generated when MGR are collected from the high seas which could be channelled into benefit-sharing. However, the same person also acknowledged that they did not think an access fee would be feasible, because it would inflict on the 'Freedom of the High Seas' principle. Therefore, from a negotiation point of view, this would likely be very difficult to implement.

\section{Discussion}

\section{A Benefit-Sharing Tool}

\section{Development of a Benefit-Sharing Methodology}

Benefit-sharing in the context of existing genetic resource frameworks under the Nagoya Protocol (conducted in a bilateral manner) is often considered to lack transparency, clarity and accountability, particularly for monetary benefit-sharing options (Fedder, 2013; Pauchard, 2017; Ruiz-Muller, 2018). For example, when dealing with ABS under the Nagoya Protocol, and since ABS contracts are private and users are not obliged to report what is in the contract, governments do not have access to information regarding the amount of money that flows out as a result of ABS. Making use of a methodology, such as that described in this study, could help to achieve a more favourable balance by negating the issue of transparency, by clarifying the key factors and helping negotiators to make informed decisions. In addition, lessons learnt from the ITPGRFA in terms of aspects to consider as part of an operating, multilateral
ABS system may also be useful here (Louafi, 2013; Stannard and Moeller, 2013; Tsioumani, 2018). Ultimately, use of this tool could help to enhance understanding and implementation of benefit-sharing policies/ legislation with greater potential to balance beneficial impacts with associated burden, thereby enhancing workability of the ABS system as a whole. However, challenges with the methodology have been identified during application in this study (as described in the section below) which should be addressed in order to develop a more robust tool for application in a variety of benefit-sharing contexts.

\section{Lessons Learnt from Application of the Methodology to the BBNJ Context}

Accurately determining the influence of different benefit-sharing options on benefit-sharing factors, such as the significance, are difficult to measure. Indeed, interviewees found it challenging to give scores for some options, partly because participants may not have fully understood or known how to measure and give an accurate answer. This is a clear aspect of the tool which could be improved in the future through consultation with a greater number of experts on that particular aspect. In addition, given the fact that these questions were asked in the context of ongoing negotiations, it is expected that some participants will have responded with strategic rather than objective answers. This may be the reason why a small minority of interviewees indicated that salaries as a form of monetary benefit-sharing would have a large positive influence on benefit-sharing factors, whilst the authors expected the impact to be moderately low compared to other options. In addition, the authors do not suggest that results collected in this study represent a comprehensive review of stakeholder perspectives on this subject matter. Further interviews with a much larger number of participants would be needed to draw significant conclusion for the BBNJ context. However, it is suggested that the benefit-sharing equation described in this article provides a useful tool and starting point, which can be built upon by decision makers in the future, to include more detailed information gathered from various expert groups, such as likely impacts on potential beneficiaries, to consolidate the perceived balance of beneficial impacts versus burden. In addition, the equation can be tailored according to different policy settings where different benefit-sharing factors and additional nuance may be more appropriate.

\section{Benefit-Sharing in the BBNJ Context}

\section{Benefit-Sharing Factors}

The most important benefit-sharing factor, according to average weights allocated by interviewees (see Figure 1), was the influence on biodiversity goals. This is likely because these goals, in particular conservation and sustainable use of biodiversity, are the primary objective of existing genetic resource frameworks, such 
as the Nagoya Protocol ${ }^{9}$ and the Plant Treaty, and as such set the precedence for similar frameworks in the future (CBD, 1992; FAO, 2001; Nagoya Protocol, 2011; World Health Organization, 2011; BBNJ, 2019a). In the BBNJ context, it has already been suggested that fair and equitable benefit-sharing related to MGR from $\mathrm{ABNJ}$ can enable the international community to address global challenges (Morgera, 2018a). These global challenges may be related to environmental protection, social objectives and private economic goals (de Brucker et al, 2013; Mohammed, 2017; Morgera, 2018a). The particular goals of greatest importance to stakeholders are different in the BBNJ context compared to areas within national jurisdiction, where aspects such as legal certainty are considered most vital (Collins et al, 2020; Sirakaya, 2020). Legal certainty is perceived to be of particularly high importance for private sector users of genetic resources (in order to promote investment), who at present appear to view genetic resources in areas within national jurisdiction as more relevant to them than MGR in ABNJ (Sirakaya, 2019; Collins et al, 2020). As such, the benefit-sharing factors involved in the methodology, and the weighting assigned to each, are likely to vary according to the circumstances.

\section{Non-Monetary Benefit-Sharing Options and Balance of Potential Positive Aspects vs Burden}

Interviewee results indicate that sharing of GSD and research results provide the most favourable balance in terms of beneficial impacts versus burden. Technology transfer, and in particular research directed towards priority needs, received less favourable balances (see Figure 3). It has been noted by the Ad Hoc Technical Expert Group on DSI on Genetic Resources under the $\mathrm{CBD}^{10}$ that DSI 'plays an important role in deepening knowledge about biodiversity, identifying and mitigating risks to threatened species, enhancing our ability to track illegal trade, identifying species and the geographic origins of products, and assisting with biodiversity planning and conservation management'. As such, the sharing of this type of information in the context of BBNJ is likely to have a positive influence on the biodiversity goals, in particular the conservation and sustainable use of BBNJ. Sharing of GSD appears to be a relatively straightforward procedure, and it is considered best practice amongst the scientific research community (Devi and Pisupati, 2018). Under the assumption that the BBNJ agreement describes requirements to share GSD in keeping with current practices, through the same current channels (and not through other systems), then this form of benefit-sharing may not incur additional burden on the users of MGR. However, the authors acknowledge the

\footnotetext{
${ }^{9}$ For example, according to Article 9 of the Nagoya Protocol (2011), 'The Parties shall encourage users and providers to direct benefits arising from the utilization of genetic resources towards the conservation of biological diversity and the sustainable use of its components'.

10 https://www.cbd.int/doc/c/079f/2dc5/2d20217d1cdacac787524d 8e/dsi-ahteg-2018-01-03-en.pdf - accessed 09 March 2020
}

contentious nature of discussions regarding GSD/DSI in multiple fora, including the CBD and the Plant Treaty. Whilst sharing of GSD/DSI itself may be relatively uncontroversial, the potential requirement for monetary benefit-sharing associated with utilisation of GSD/DSI is highly contentious (Kobayashi, 2019). Sharing of research results is thought to have a similar positive effect to the sharing of GSD on enhancing the potential for conservation of marine biodiversity, by helping to build an enabling environment in which MSR can flourish (Harden-Davies and Gjerde, 2019).

Technology transfer represented a relatively unfavourable balance. This may be associated with a combination of relatively high expected burden on the users of genetic resources and on the regulators, together with limited potential beneficial impacts. The potential burdens on different stakeholder groups associated with technology transfer are described in Collins et al (2020). It is suggested that the degree of burden, in terms of financial cost and administration, will depend on the type of technology, the conditions, how it is funded and how the transfer is managed, but on the whole, these will be more significant than for other forms of non-monetary benefit-sharing. For example, it is generally expected that the funding required for technology transfer will be greater than for other nonmonetary benefit-sharing options (Collins et al, 2020). In addition, the owners of technology are often private companies or individuals (Prip et al, 2015). As such, transfer of this technology will need to involve consideration of commercial aspects, such as the inherent requirement for private sector entities to generate a return on investment and maintain market competitiveness. Related factors will include appropriate intellectual property arrangements and/ or private economic incentives or public funding, particularly in the circumstance of private ownership of technology (Prip et al, 2015). Moreover, the beneficial impacts linked to technology transfer may be limited by absorptive capacity (or enabling factors) in the recipient state. According to Prip et al (2015), successful technology transfer depends on three factors: the existence of relevant technology to address particular (environmental) challenges, the relevant dissemination of technology in a manner which makes it available to entities which need this, and the application of technology in a manner which is conducive to solving the challenges in mind.

As noted by interviewees involved in a study conducted by Sirakaya (2019), research directed towards priority needs would involve a degree of burden associated with making sure that the research precisely benefits the provider country (or in the context of BBNJ, the beneficiary state(s)), because this would require a considerable amount of time, effort and resources. This potential burden is likely to contribute to the less favourable balance for research directed towards priority needs. 


\section{Monetary Benefit-Sharing Options and Balance of Potential Positive Aspects vs Burden}

According to interviewee results, research funding, followed closely by salaries, were selected as the monetary benefit-sharing options which provide the most favourable balance regarding beneficial impacts versus burden. Access fees, milestone payments, license fees and royalties received less favourable balances (see Figure 4).

The favourable benefit-sharing balance indicated for research funding and salaries may be because there is a greater degree of perceived transparency involved in these approaches, compared to other forms of monetary benefit-sharing, in terms of where the financial resources are directed and how 'beneficial' this could be (Altman and Simera, 2010). This transparency with regards to research funding is promoted by funding organisations $^{11,12}$, science foundations ${ }^{13}$, journals and international initiatives, such as the EQUATOR (Enhancing the QUAlity and Transparency Of health Research) network, which aim to encourage transparent reporting of research, research funding processes and the use of reporting guidelines ${ }^{14}$. In addition, salaries and in particular research funding can directly support R\&D on MGR, with potential to enhance conservation and sustainable use of BBNJ.

The less favourable balance indicated for access fees, milestone payments, license fees and royalties may, therefore, be associated with a lack of clarity and transparency in terms of how financial resources are used and how much of a beneficial impact this might have (Altman and Simera, 2010). Indeed, interviewees involved in this study raised questions and uncertainty regarding how money could be utilised in the scope of monetary benefit-sharing options to promote biodiversity goals, such as conservation and sustainable use of biodiversity. This uncertainty may also be due to the complicated nature of the factors explored. Further research, building on the current study, should be more specific in nature and would likely be simpler for interviewees to respond to, yielding increasingly insightful results. For example, it was suggested that the balance for monetary benefit-sharing options could be more favourable than currently perceived if money could clearly be linked to supporting these goals. Whilst some monetary benefit-sharing options may at some stage create incentives for biodiversity conservation, this would depend on how and where financial resources are allocated. This is currently not as clear or as transparent as processes associated with research funding and salaries (Altman and Simera, 2010). In addition, the potential impact of benefit-sharing options

\footnotetext{
11 https://www.nihr.ac.uk/blog/how-do-you-make-research-fundingtransparent-and-fair/10991 - accessed 02 March 2020

12 https://www.ukri.org/about-us/policies-and-standards/transparen cy/ - accessed 09 March 2020

13 https://www.nsf.gov/od/transparency/transparency.jsp - accessed 21 March 2020

14 https://www.equator-network.org/- accessed 09 March 2020
}

on bioidiversity goals and the overall benefit-sharing balance may be affected by the approach and language adopted in the new agreement. For example, the Nagoya Protocol (2011) text refers mostly to 'encouraging' the flow of benefits towards biodiversity conservation, but there is no clear obligation ${ }^{15}$. The Benefit-Sharing Fund of the Plant Treaty provides a different example, whereby the Contracting Parties decide for themselves the contributions that they will make (Tsioumani, 2018). In order to reach a more favourable balance, it will be necessary to include language in the BBNJ agreement which clearly ties benefit-sharing options to the objectives. This is an important point which should be kept in mind during the negotiation process.

In this study, the authors investigated benefit-sharing options in the manner in which they are currently being addressed in accordance with existing ABS frameworks under the Nagoya Protocol, Plant Treaty and in the draft BBNJ text. When investigating details regarding monetary benefit-sharing options with a view to developing a methodology to balance these with various factors, it became apparent that these options are pooled together under the 'monetary benefitsharing' term without making the distinction between those which accumulate (such as royalties, access fees and milestone payments) and those which distribute funding (such as research funding and salaries). Given that monetary benefit-sharing is already a contentious item in the realm of benefit-sharing, the fact that it is dealt with in this confusing manner is not useful and could prove problematic when translated into practice. This point represents an important finding in the present study. Whilst out of scope of this paper, further research should be conducted in the future to adjust and tailor the formula according to whether the monetary benefitsharing options generate or distribute funding.

A further factor which might contribute towards the less favourable balance of monetary benefit-sharing options compared to the non-monetary options is the fact that the benefits will need to be shared amongst large numbers of beneficiaries. Whilst sharing of data or information with many people does not dilute or divide the benefits received by each individual/ entity (each can receive the same package of data/ information), the sharing of monetary benefits amongst many beneficiaries will likely result in limited and small amounts of money reaching the beneficiaries. This aspect may contribute towards the perception held by many delegates that non-monetary benefitsharing could represent more predictable and more significant options than forms of monetary benefitsharing (Morgera, 2018b). By focusing on goals and identified capacity requirements, non-monetary benefitsharing can have a more immediate and tangible impact

\footnotetext{
${ }^{15}$ Article 9, Nagoya Protocol, 2011: 'The Parties shall encourage users and providers to direct benefits arising from the utilization of genetic resources towards the conservation of biological diversity and the sustainable use of its components.'
} 
on bridging the equity gaps related to R\&D on MGR. This approach has been considered the principal success of the Plant Treaty (Stannard and Moeller, 2013; Tsioumani, 2018).

\section{Conclusion}

To date, benefit-sharing related to the utilisation of genetic resources has not been as effective as was hoped (Ruiz-Muller, 2018; Pauchard, 2017). This is at least partially related to the lack of focus associated with benefit-sharing options on conservation and sustainable use of biodiversity, possible hampering of scientific research as well as the burden placed on the regulator and users of genetic resources. This has in some cases led to a loss of incentive to conduct R\&D on genetic resources, with potential to further limit conservation and sustainable use of biodiversity (Tvedt, 2013). The authors acknowledge that there are many other political and legal issues, in addition to the technical aspects referred to in this study, which contribute towards the challenges currently experienced with existing benefitsharing systems. These issues include, for example, the lack of objectivity in terms of approaches to agreeing appropriate benefit-sharing measures.

A tool, such as the methodology presented in this study (Figure 1), provides a useful means to assess the balance of different benefit-sharing options in terms of the potential beneficial and burdensome impacts, taking into account the varying relative importance of different factors. This would help policy-makers in the future when deciding which forms of benefit-sharing may be most appropriate to adopt, or when improving existing ABS measures, with greater potential for meeting proposed objectives, such as conservation and sustainable use of biodiversity. Use of this methodology would also likely promote transparency, objectivity, clarity and workability of the benefit-sharing system, possibly leading to enhanced generation, accrual and sharing of benefits (Lindhjem et al, 2010).

Results in this study indicate that in terms of nonmonetary benefit-sharing options under consideration for the new BBNJ agreement, sharing of GSD and research results may provide the most favourable balance. Should monetary benefit-sharing be included in the agreement, research funding and salaries may represent the most fairly balanced options. In addition, in order to reach a favourable balance, it may be necessary to include language in the BBNJ agreement which clearly ties benefit-sharing options to the objectives. However, further interviews with a larger number of participants would be needed to draw significant conclusion for the BBNJ context. Nonetheless, the benefit-sharing equation described and demonstrated in this article provides a useful tool and starting point, which can be tailored according to different policy settings where consideration of different benefit-sharing factors may be more appropriate. In addition, the methodology can be developed to include more detailed information gathered from various expert groups to consolidate the perceived balance of beneficial impacts versus burden associated with benefit-sharing options.

\section{Acknowledgements}

Many thanks to all of the interviewees for their valuable contribution towards this research. Thanks also to the Marie Curie H2020 fund (H2020-MSCA-ITN-ETN: MarPipe GA 721421) for supporting this research.

\section{Supplemental data}

Supplemental File 1: Outline for interviews for "Developing a Methodology to Balance Benefit-Sharing: Application in the Context of Biodiversity Beyond National Jurisdiction".

\section{Author contributions}

JC and TV jointly conceived the idea for the study and manuscript. JC developed the research methodology, performed data collection, data analysis and led writing and editing of the text. AS assisted with developing the research methodology and co-wrote and edited the manuscript. TV also assisted with developing the research methodology and edited and reviewed the manuscript. IH edited and reviewed the manuscript.

\section{Conflict of interest statement}

The authors declare no conflicts of interest.

\section{References}

Altman, D. G. and Simera, I. (2010). Responsible reporting of health research studies: transparent, complete, accurate and timely. Journal of Antimicrobial Chemotherapy 65(1), 1-3. url: https://doi.org/ 10.1093/jac/dkp410.

BBNJ (2019a). Draft text of an agreement under the United Nations Convention on the Law of the Sea on the conservation and sustainable use of marine biological diversity of areas beyond national jurisdiction. In Intergovernmental Conference on an International Legally Binding Instrument Under the United Nations Convention on the Law of the Sea on the Conservation and Sustainable Use of Marine Biological Diversity of Areas Beyond National Jurisdiction Third Session (New York, NY). https:/ /undocs.org/a/conf.232/2019/6 (accessed 06 March 2020).

BBNJ (2019b). Revised draft text of an agreement under the United Nations Convention on the Law of the Sea on the conservation and sustainable use of marine biological diversity of areas beyond national jurisdiction. In and others, Intergovernmental Conference on an International Legally Binding Instrument Under the United Nations Convention on the Law of the Sea on the Conservation and Sustainable Use of Marine Biological Diversity of Areas Beyond National Jurisdiction Fourth Session. 
https://undocs.org/a/conf.232/2019/6 (accessed 06 March 2020).

CBD (1992). Convention on Biological Diversity, opened for signature 5 June 1992, 1760 UNTS 79. (entered into force 29 December 1993).

Collins, J. E., Vanagt, T., and Huys, I. (2020). Stakeholder Perspectives on Access and BenefitSharing for Areas Beyond National Jurisdiction. Frontiers in Marine Science 7, 265-265. url: https: //doi.org/10.3389/fmars.2020.00265.

Correa, C. M. (2017). Access to and benefit-sharing of marine genetic resources beyond national jurisdiction: Developing a new legally binding instrument. In Routledge handbook of biodiversity and the law, ed. McManis, C. R. and Ong, B. (Routledge), 157-174.

de Brucker, K., Macharis, C., and Verbeke, A. (2013). Multi-criteria analysis and the resolution of sustainable development dilemmas: A stakeholder management approach. European journal of operational research 224(1), 122-131. url: https://doi.org/10. 1016/j.ejor.2012.02.021.

de Jonge, B. (2009). Plants, genes and justice: an inquiry into fair and equitable benefit-sharing. Ph.D. thesis, Wageningen University, Wageningen University, Wageningen, The Netherlands. ISBN 97890-8585-472-2.

de Jonge, B. (2011). What is fair and equitable benefitsharing? J Agric Environ Ethics 24(2), 127-146. url: https://doi.org/10.1007/s10806-010-9249-3.

Devi, S. and Pisupati, B. (2018). Open Source DNA Data, Information and the Nagoya Protocol. url: http://fledgein.org/wp-content/uploads/ 2018/07/FINAL-DSI-paper.pdf.

Dodgson, J. S., Spackman, M., Pearman, A., and Phillips, L. D. (2009). Multi-criteria analysis: a manual. url: http://eprints.lse.ac.uk/12761/1/Multicriteria_Analysis.pdf. accessed date: 20200309

FAO (2001). International Treaty on Plant Genetic Resources for Food and Agriculture. FAO Res. 3/2001, 3 November 2001 (entered into force 29 June 2004). url: http://www.fao.org/3/a-i0510e.pdf.

Fedder, B. (2013). Marine Genetic Resources, Access and Benefit Sharing: Legal and Biological Perspectives (Routledge). url: https://doi.org/10.4324/ 9780203381618.

Freeman, R. E. (1984). Strategic Management: A Stakeholder Approach (Boston: Pitman). url: https: //doi.org/10.1017/CBO9781139192675.

Harden-Davies, H. R. and Gjerde, K. M. (2019). Building Scientific and Technological Capacity: a Role for Benefit-sharing in the Conservation and Sustainable Use of Marine Biodiversity beyond National Jurisdiction. Ocean Yearbook Online 33(1), 377-400. url: https://doi.org/10.1163/9789004395633_015.

Ho, W., Xu, X., and Dey, P. K. (2010). Multi-criteria decision making approaches for supplier evaluation and selection: A literature review. European Journal of Operational Research 202(1), 16-24. url: https:// doi.org/10.1016/j.ejor.2009.05.009.
Kobayashi, K. (2019). Will the Discussion on Digital Sequence Information on Genetic Resources Contribute to the" Evolution" of CBD? Biodiversity Information Science and Standards 3, e36593. url: https://doi.org/10.3897/biss.3.36593.

Lindhjem, H., Aronsen, I., Br, K. G., and Gleinsvik, A. (2010). Experiences with benefit sharing: issues and options for REDD-plus. Commissioned by International Union for Conservation of Nature (IUCN) .

Louafi, S. (2013). Reflections on the resource allocation strategy of the Benefit Sharing Fund: Policy Brief.

Mohammed, E. (2017). Governing the high seas: priorities for the Least Developed Countries. IIED Briefing Papers. url: https://pubs.iied.org/16634IIED/.

Morgera, E. (2014). Conceptualizing benefit-sharing as the pursuit of equity in addressing global environmental challenges. BENELEX Working Paper 1, Edinburgh School of Law Research Paper No. 2014/41. url: http://dx.doi.org/10.2139/ssrn.2524003.

Morgera, E. (2018a). Equity and benefit sharing from marine genetic resources in areas beyond national jurisdiction. IIED Briefing Papers. url: https://pubs. iied.org/17462IIED/.

Morgera, E. (2018b). Fair and equitable benefit-sharing in a new treaty on marine biodiversity: a principled approach towards partnership building. Maritime Safety and Security Law Journal (5), 48-77.

Morgera, E. and Tsioumani, E. (2010). The Evolution of Benefit Sharing: Linking Biodiversity and Community Livelihoods. Review of European Community \& International Environmental Law 19(2), 150-173. url: https: //dx.doi.org/10.1111/j.1467-9388.2010.00674.x.

Nagoya Protocol (2011). Nagoya protocol on access to genetic resources and the fair and equitable sharing of benefits arising from their utilization to the convention on biological diversity. url: https://www. cbd.int/abs/text/.

Nerini, F. F., Howells, M., Bazilian, M., and Gomez, M. F. (2014). Rural electrification options in the Brazilian Amazon. Energy for Sustainable Development 20, 3648. url: https://doi.org/10.1016/j.esd.2014.02.005.

Parks, L. (2019). Benefit-sharing in Environmental Governance (Open Access): Local Experiences of a Global Concept. (Routledge).

Pauchard, N. (2017). Access and Benefit Sharing under the Convention on Biological Diversity and Its Protocol: What Can Some Numbers Tell Us about the Effectiveness of the Regulatory Regime? Resources 6, 11. url: https://doi.org/10.3390/resources6010011.

Pisupati, B. and Bavikatte, S. K. (2014). Access and benefit sharing as an innovative financing mechanism. Asian Biotechnol Dev Rev 16(2), 53-70.

President's aid to negotiations (2019). Intergovernmental conference on an international legally binding instrument under the United Nations Convention on the Law of the Sea on the conservation and sustainable use of marine biological diversity of areas beyond national jurisdiction. Second session. In Proceedings 
of the Intergovernmental Conference On Marine Biodiversity Of Areas Beyond National Jurisdiction. url: https://undocs.org/A/CONF.232/2019/1 (accessed 06 March 2020).

Prip, C., Rosendal, G. K., and Tvedt, M. W. (2015). The state of technology transfer obligations in global environmental governance and law: biodiversity conservation and sustainable use. FNI Report 4/2015, 1-18. url: https://doi.org/10.13140/RG.2.2.14108. 28804.

Richerzhagen, C. and Holm-Mueller, K. (2005). The effectiveness of access and benefit sharing in Costa Rica: implications for national and international regimes. Ecological Economics 53(4), 445-460. url: https://doi.org/10.1016/j.ecolecon.2004.06.031.

Ruiz-Muller, M. (2018). Access to Genetic Resources and Benefit Sharing 25 Years on: Progress and Challenges. International Centre for Trade and Sustainable Development (ICTSD), Geneva, 36p.

Schroeder, D. (2007). Benefit sharing: it's time for a definition. Journal of Medical Ethics 33(4), 205-209. url: https://dx.doi.org/10.1136/jme.2006.016790.

Sirakaya, A. (2019). Balanced Options for Access and Benefit-Sharing: Stakeholder Insights on Provider Country Legislation. Frontiers in Plant Science 10, 1175. url: https://doi.org/10.3389/fpls.2019.01175.

Sirakaya, A. (2020). A balanced ABS system: Stakeholder perception on ABS goals. Sustainable Development 28(3), 495-503. url: https://doi.org/10. 1002/sd.2040.

Stannard, C. and Moeller, N. I. (2013). Identifying benefit flows: studies on the potential monetary and non-monetary benefits arising from the international treaty on plant genetic resources for food and agriculture (Rome: FAO), 315p. url: https://www.bioversityinternational.org/e-library/publications/detail/identifying-benefit-flowsstudies-on-the-potential-monetary-and-non-monetarybenefits-arising-from- $t$ /.
Suneetha, M. S. and Pisupati, B. (2009). Benefit sharing in abs: Options and elaborations (UNU-IAS Report). url: https://www.cbd.int/abs/doc/unu-absreport-2009-en.pdf.

Tsioumani, E. (2018). Beyond access and benefitsharing: Lessons from the emergence and application of the principle of fair and equitable benefit-sharing in agrobiodiversity governance. In The Commons, Plant Breeding and Agricultural Research, (Routledge). 41-60.

Tvedt, M. W. (2013). Beyond Nagoya: towards a legally functional system of access and benefit sharing. In Global Governance of Genetic Resources: Access and Benefit Sharing after the Nagoya Protocol, ed. Oberthür, S. and Rosendal, G. K. (Routledge), 158-177.

UNCLOS (1982). United Nations Convention on the Law of the Sea. opened for signature 10 December 1982, 1833 UNTS 3 (entered into force 16 November 1994).

Voigt-Hanssen, G. (2018). C Current 'Light' and 'Heavy' Options for Benefit-sharing in the Context of the United Nations Convention on the Law of the Sea. The International Journal of Marine and Coastal Law 33(4), 683-705. url: https://doi.org/10.1163/ 15718085-12334012.

von Winterfeldt, D. and Edwards, W. (1986). Decision Analysis and Behavioral Research (New York: Cambridge University Press).

World Health Organization (2011). Pandemic influenza preparedness (PIP) framework for the sharing of influenza viruses and access to vaccines and other benefits. url: http://www.who.int/influenza/pip/en.

Young, T. R. and Tvedt, M. W. (2017). Drafting Successful Access and Benefit-sharing Contracts (Brill). url: https://doi.org/10.1163/9789004356573. 